\title{
A Disulfide Bridge Mediated at Cysteine 574 Is Formed in the Dimer of the 70-kDa Heat Shock Protein
}

Takayuki K. Nemoto, ${ }^{1}$ Yutaka Fukuma, ${ }^{1}$ Hideaki Itoh, ${ }^{2}$ Takashi Takagi ${ }^{3}$ and Toshio Ono ${ }^{1}$

${ }^{I}$ Division of Oral Molecular Biology, Department of Developmental and Reconstructive

Medicine, Course of Medical and Dental Sciences, Nagasaki University Graduate

School of Biomedical Sciences, 1-7-1 Sakamoto, Nagasaki 852-8588, Japan

${ }^{2}$ Department of Material-Process Engineering and Applied Chemistry for Environment, Akita University Faculty of Engineering and Resource Science, 1-1 Tegata Gakuen Town, Akita City 010-8502, Japan

${ }^{3}$ Department of Developmental Biology and Neurosciences, Graduate School of Life Sciences, Tohoku University, Sendai 980-8578, Japan

Key words: Hsp70, disulfide bridge, dimer, lid, client-binding

Running head: Dimeric structure of Hsp70

*To whom correspondence should be addressed. Tel: +81-95-849-7640, Fax: +81-95-849-7642;

E-mail: tnemoto@net.nagasaki-u.ac.jp 
Abbreviations: Hsp, heat shock protein; Hsp70, inducible 70-kDa heat shock protein; Hsc70, 70-kDa heat shock cognate protein; Hsp90, 90-kDa heat shock protein; Hsp60/GroEL 60-kDa heat shock protein; mAb, monoclonal antibody; PAGE, polyacrylamide gel electrophoresis; SDS, sodium dodecylsulfate; and TPCK, $\mathrm{N}^{\alpha}$-acetyl-L-tosyl-L-phenylalanine chloromethyl ketone. 
The $70-\mathrm{kDa}$ heat shock protein (Hsp70) is predominantly present intracellularly as a monomer, but a small population is converted to dimers and oligomers under certain conditions. In the present study, we investigated the dimeric structure of human inducible Hsp70. As reported earlier, the C-terminal client-binding domain (amino acids 382-641) was required for the dimerization. A 40-amino acid deletion of the client-binding domain from either the $\mathrm{N}$-terminus or $\mathrm{C}$-terminus greatly enhanced the dimerization potential of Hsp70. Limited proteolysis indicated that the dimer formed by truncation from the C-terminus had a conformation similar to that of the non-truncated form. Truncation experiments demonstrated that the client-binding sub-domain (amino acids 382-520) with its adjacent region up to amino acid 541 was not sufficient for the dimerization but that the region up to amino acid 561 was sufficient. Interestingly, the dimer formed by truncation from the C-terminus acquired a homomeric disulfide bridge at Cys574. 
Heat shock proteins (Hsps) exist essentially in all organisms. Reflecting their important and general roles as molecular chaperones, their amino acid sequences are highly conserved in every Hsp family even among eukaryotic and prokaryotic organisms. Among the 3 major Hsps in the high-molecular-weight class, i.e., 60-kDa, 70-kDa and 90-kDa heat shock proteins (Hsp60/GroEL, Hsp70 and Hsp90, respectively), the architecture of Hsp60/GroEL oligomers complexed with Hsp10/GroES has been extensively investigated (1). Hsp90 forms a dimer mediated through its C-terminal region (2-3). Based on the results of X-ray crystallographic studies (4-7), Prodromou et al. $(4,5)$ and Meyer et al. (7) proposed the molecular clamp model, in which a dimer of Hsp90 traps client proteins between the N-terminal domains or middle domains. On the other hand, Hsp70 predominantly exists as a monomer (8). Nevertheless, it has also been reported that Hsp70 and DnaK, a bacterial ortholog of Hsp70, are dimerized and further oligomerized in a concentration dependent manner (9). Moreover, ATP induces the dissociation of dimers and oligomers of Hsp70 (9-12). Therefore, it has been suggested that the monomer-dimer conversion is closely related to the chaperone function of Hsp70.

Hsp70 is composed of at least 2 structural domains (13). The 44-kDa N-terminal domain of bovine brain Hsp70 contains a nucleotide-binding site and possesses a weak ATP-hydrolytic activity (13). The 30-kDa C-terminal domain of DnaK has the capacity to bind unfolded polypeptides $(14,15)$. Although the 3-dimensional structure of the full-length forms of Hsp70 remains unknown, it is obvious that the activities of these domains are co-operative, because the client-binding activity of Hsp70 is relatively high in the ADP-bound state, but is reduced in the ATP-binding state $(9,16)$ and the binding of client peptides leads to the stimulation of ATP hydrolysis by $\operatorname{Hsp} 70(17,18)$.

There is some evidence that the $30-\mathrm{kDa}$ C-terminal client-binding domain is responsible for the dimerization of $70-\mathrm{kDa}$ heat shock cognate protein $\operatorname{Hsc} 70(19,20)$. It has been reported that the 30-kDa C-terminal domain can be divided into N-terminal 18-kDa and C-terminal 10-kDa sub-domains. The N-terminal sub-domain (designated as the client-binding sub-domain) contains the binding site for client peptides (21), and the C-terminal sub-domain (designated as the lid sub-domain) appears to function as a lid that stabilizes the client peptide bound to the client-binding sub-domain. Fouchaq et al. (20) reported that the 17-kDa client-binding sub-domain of Hsc70 could be oligomerized. 
In contrast, Chou et al. (22) proposed that the 10-kDa lid sub-domain of Hsc70 is responsible for the dimerization and oligomerization. Therefore, it remains unknown as to whether the client-binding sub-domain or the lid sub-domain is critical for the dimerization.

In the present study, we expressed the full-length form of human Hsp70 (hHsp70), the C-terminal client-binding domain and their truncated forms to examine the region responsible for the dimerization and oligomerization of Hsp70. We found that several forms of the truncated C-terminal domain acquired a dimerization potential that was much higher than that of the client-binding domain itself or the full-length form. Unexpectedly, some of the dimeric complexes possessed disulfide bridge(s) connecting Cys574 of each molecule. 


\section{EXPERIMEMTAL PROCEDURES}

Materials - The materials used and their sources were the following: Expression vector pTrcHis TOPO and TOP10 competent cells, from Invitrogen (Carlsbad, CA, USA); Low-molecular-weight markers and peptide markers, from Amersham Biosciences (Piscataway, NJ, USA); kaleidoscope prestained molecular standard, from Bio-Rad (Richmond, CA, USA); restriction enzymes and DNA-modifying enzymes, from Nippon Gene (Tokyo, Japan); Talon metal affinity resin, from Clontech Laboratories Inc. (Palo Alto, CA, USA); trypsin (5,200 USP units/mg protein) and $\mathrm{N}^{\alpha}$-acetyl-L-tosyl-L-phenylalanine chloromethyl ketone (TPCK) from Sigma (St. Louis, MO, USA.); anti-histidine hexamer tag monoclonal antibody (anti-His tag $\mathrm{mAb}$ ) from Qiagen Inc. (Chatworth, CA, USA); and alkaline-phosphatase-conjugated rabbit anti-mouse $\operatorname{Ig}(\mathrm{G}+\mathrm{A}+\mathrm{M})$ antibody from Zymed Laboratories Inc. (San Francisco, CA, USA).

Construction of bacterial expression plasmids - All recombinant proteins used in this study were encoded by pTrcHis TOPO vector (Invitrogen), and therefore, possessed a 35-amino acid tag carrying a histidine-hexamer at the N-terminus. cDNA encoding the full-length form of hHsp70 was obtained by RT-PCR from HeLa cell mRNA with the aid of its cDNA sequence $(23,24)$. For construction of the plasmids carrying the full-length form of hHsp70, the ATP-binding domain (amino acids 1-381), the client-binding domain (amino acids 382-641) and truncated forms of the client-binding domain (Fig. 1), DNA fragments encoding the corresponding regions were amplified by PCR, and then directly inserted into the pTrcHis TOPO expression vector in frame according to the manufacturer's recommendation. TOP10 cells were transformed with the plasmids and selected on Luria-Bertani medium-agar plates containing $50 \mu \mathrm{g} / \mathrm{ml}$ of ampicillin. The insertion and orientation of the DNA fragments were confirmed by conducting Hot Star PCR (Qiagen Inc., Chatsworth, CA, USA).

Expression and purification of recombinant proteins - After overnight cultivation of transformed bacteria at $37^{\circ} \mathrm{C}$, recombinant proteins were expressed at $30^{\circ} \mathrm{C}$ for $4 \mathrm{~h}$ in the presence of $0.2 \mathrm{mM}$ isopropyl- $\beta$-D-thiogalactopyranoside. They were purified by affinity chromatography with a Talon affinity column according to the manufacturer's protocol except that $10 \mathrm{mM}$ imidazole and $10 \mu \mathrm{g} / \mathrm{ml}$ of 
leupeptin were added to the lysis buffer $(20 \mathrm{mM}$ Tris- $\mathrm{HCl}, \mathrm{pH} 8.0$, and $0.1 \mathrm{M} \mathrm{NaCl}$ containing 0.5 $\mathrm{mg} / \mathrm{ml}$ lysozyme). After extensive washing with washing buffer $(20 \mathrm{mM}$ Tris-HCl, $\mathrm{pH} 8.0$, containing $0.1 \mathrm{M} \mathrm{NaCl}$ and $10 \mathrm{mM}$ imidazole) to remove non-adsorbed proteins, bound proteins were eluted with $0.1 \mathrm{M}$ imidazole $(\mathrm{pH}$ 8.0) containing $10 \%(\mathrm{v} / \mathrm{v})$ glycerol. Purified proteins were used immediately or stored at $-80^{\circ} \mathrm{C}$ until used.

Sodium dodecyl sulfate-polyacrylamide gel electrophoresis (SDS-PAGE) - Electrophoresis was performed in the presence of $0.1 \%(\mathrm{w} / \mathrm{v})$ SDS according to Laemmli (25). Unless otherwise stated, a $12.5 \%(\mathrm{w} / \mathrm{v})$ polyacrylamide concentration was used. For separation of small molecular species such as proteolytic fragments, SDS-PAGE by the Tris-Tricine system at a $10 \%(\mathrm{w} / \mathrm{v})$ polyacrylamide concentration was employed (26). Samples were denatured at $94^{\circ} \mathrm{C}$ for $10 \mathrm{~min}$ in the sample buffer in the presence or absence of $10 \%(\mathrm{v} / \mathrm{v})$ 2-mercaptoethanol. Low-molecular-weight markers (Amersham Biosciences, Piscataway, NJ, USA) and peptide markers (Amersham Biosciences) were used as references.

Polyacrylamide gel electrophoresis under nondenaturing conditions - In order to estimate molecular configurations, samples were subjected to PAGE under nondenaturing conditions (27). Unless otherwise stated, a $7.5 \%(\mathrm{w} / \mathrm{v})$ polyacrylamide concentration was used. Electrophoresis was performed at low voltage $(50 \mathrm{~V})$ at room temperature to avoid elevation of the gel temperature. Separated proteins were stained with Coomassie brilliant blue. Ovalbumin (45 kDa), bovine serum albumin (66 kDa as monomer and $132 \mathrm{kDa}$ as dimer) and catalase (240 kDa) were used as molecular references.

Two-dimensional PAGE - Purified proteins were separated by 2-dimensional PAGE. A 7.5 \%(w/v) polyacrylamide concentration was used at the first dimension PAGE under non-denaturing conditions and an $11 \%(\mathrm{w} / \mathrm{v})$ concentration was used at the second dimension SDS-PAGE under reducing or non-reducing conditions. 
Immunoblotting analysis - Recombinant proteins in the bacterial lysates separated by PAGE under non-denaturing conditions were subjected to the immunoblotting analysis as described previously (28). An anti-histidine tag mAb (Qiagen) was used as the first antibody at $1 \mu \mathrm{g} / \mathrm{ml}$, and the second antibody was used at a 1/3000 dilution. Blotted proteins were finally visualized by incubation with 5-bromo-4-chloro-3-indolyl phosphate and nitro blue tetrazolium (Nakarai, Kyoto, Japan) at room temperature for 10-20 $\mathrm{min}$.

Size-exclusion gel chromatography - Size-exclusion gel chromatography was performed on a Sephacryl S100HR (Amersham Biosciences, 1.8 x 36 cm) or Sephacryl S300 (Amersham Biosciences, 1.0 x $90 \mathrm{~cm}$ ) column equilibrated with $20 \mathrm{mM}$ Tris- $\mathrm{HCl}(\mathrm{pH} 8.0)$ containing $1 \mathrm{mM}$ EDTA, $50 \mathrm{mM}$ $\mathrm{NaCl}$ and $0.05 \%(\mathrm{w} / \mathrm{v}) \mathrm{NaN}_{3}$. Blue dextran 2000 (void volume), catalase (232 kDa, Stokes radius $=52.2 \mathrm{~A})$, aldolase $(158 \mathrm{kDa}, 48.1 \mathrm{~A})$, bovine serum albumin $(66 \mathrm{kDa}, 35.5 \mathrm{~A})$ and ovalbumin $(45$ $\mathrm{kDa}, 30.5 \mathrm{~A}$ ) were used as molecular size references. Forty-drop fractions ( $2 \mathrm{ml})$ were collected from the Sephacryl S100HR column; and 20-drop fractions (1 ml), from the Sephacryl S300 column.

$N$-terminal amino acid sequencing - In order to investigate the difference in molecular configuration, we partially cleaved the client-binding domain and its truncated forms with trypsin treated with TPCK. Recombinant proteins in the elution buffer were incubated at $30^{\circ} \mathrm{C}$ with $3 \mathrm{ng}$ of TPCK-trypsin for 0-12 h or with 0-300 ng of TPCK-trypsin at $30^{\circ} \mathrm{C}$ for $12 \mathrm{~h}$. Following electrophoretic separation of proteolytic fragments by use of the Tris-Tricine system, major tryptic fragments were transferred to a polyvinylidene difluoride membrane (Bio-Rad). After having been stained with Coomassie brilliant blue, the bands were excised and directly sequenced with a model 492A protein sequencer (PE Biosystems).

Protein concentration - Protein concentrations were determined by the bicinchoninic acid method using bovine serum albumin as a standard (Pierce, Rockford, IL, USA). 


\section{RESULTS}

Dimerization of the full-length form of $h H s p 70$ - Initially we expressed the full-length form of hHsp70 in a bacterial expression system. Upon SDS-PAGE under reducing conditions (Fig. 1a, right, lanes 1-4), the purified protein migrated as a single band at the position of $75 \mathrm{kDa}$, which was slightly larger than the value of endogenous hHsp70 (calculated molecular mass of 70,053; ref 23) due to the 35 -amino acid tag at the $\mathrm{N}$-terminus.

Even if large amounts of the purified sample were loaded onto an SDS-PAGE gel, no additional band appeared under reducing conditions (Fig. 1a, right). In contrast, under non-reducing conditions, additional bands appeared at positions indicating high molecular masses (Fig. 1a, left, lanes 3 and 4). Among them, the most predominant band migrated to a $150-\mathrm{kDa}$ position, indicating the covalently-associated dimeric configuration of 75-kDa hHsp70.

Polyacrylamide gel electrophoresis under non-denaturing conditions revealed one major band (Fig. 1b, lanes 1 and 2), and a second, slow-migrating species appeared at higher protein concentrations (lanes 3 and 4). At the highest protein concentration, a third band appeared (lane 4). Taken the migrating position and the results of previous studies $(11,12)$ in mind, the 3 bands may correspond to the monomer, dimer and trimer.

We noticed that, the bands presumably corresponding to the monomer, dimer and trimer of hHsp70 migrated slower than expected, when compared with the molecular mass references (bovine serum albumin, ovalbumin and catalase) used in this study. This discrepancy seemed to be due to the molecular size references used for PAGE under non-denaturing conditions, because the isoelectric point $(\mathrm{pI})$ of $\mathrm{Hsp} 70(\mathrm{pI}=5.5-5.6$; ref 8$)$ is more basic than that of ovalbumin $(\mathrm{pI}=4.6)$ or bovine serum albumin ( $\mathrm{pI}=4.8 ;$ ref 27$)$.

Charge properties of hHsp70 affecting on the electrophoretic mobility on PAGE under non-denaturing conditions - Net charges of the N-terminal ATP-binding and C-terminal client-binding domains simply calculated from the amino acid composition are -1 and -10 , respectively (24). Hence, we suspected that the slow migration of $\mathrm{hHsp} 70$ was primarily caused by the charge property of the N-terminal ATP-binding domain. To prove this hypothesis, we 
expressed the N-terminal ATP-binding (amino acids 1-381) and C-terminal client-binding domains (amino acids 382-641) and 2 sub-domains (amino acids 382-519 and 520-641) of the client-binding domain (Fig. 2a). Upon SDS-PAGE (Fig. 2b, left), they migrated as a single band with an exception that the lid sub-domain (hHsp70 520-641), which contained a 22-kDa species as a minor constituent (lane 5). The migration of hHsp70 1-381 was much slower than that of hHsp70 382-641 (Fig. 2b, right, compare lanes 2 and 3). This retardation of hHsp70 1-381 should reflect either the dimerization or the charge property. To discriminate these 2 possibilities, hHsp70 1-381 was separated by size exclusion gel chromatography on a Sephacryl S100HR column. As a result, hHsp70 1-381 eluted as a single peak with a molecular size smaller that 45-kDa ovalbumin (Fig. 2c). Therefore, we concluded that hHsp70 was monomeric, but migrated slowly on PAGE under non-denaturing conditions because of its less acidic charge property. To the contrary, the main band of hHsp70 382-641 migrated fast on PAGE under non-denaturing conditions, reflecting its acidic property (Fig. 2b, right, lane 3).

Dimerization of the C-terminal client-binding domain - Polyacrylamide gel electrophoresis under non-denaturing conditions revealed the presence of slow-migrating species in the preparations of hHsp70 382-641 and 520-641 in addition to respective fast-migrating major ones (Fig. 2b, right, arrowheads). We suspected that the fast- and slow-migrating species corresponded to the monomer and dimer, respectively. In fact, 2-dimensional PAGE of hHsp70 382-641 confirmed the existence of monomeric, dimeric, trimeric and presumably oligomeric species (Fig. 2d). In contrast, a slow-migrating species of hHsp70 520-641 was not a dimer, but corresponded to the 22-kDa band, which was co-purified with the major 24-kDa band (Fig. 2e, asterisks). The 22-kDa species seemed to be a degraded product of the 24-kDa hHsp70 520-641. These findings confirmed the results of the previous study (19) demonstrating that the dimerization as well as the oligomerization of Hsp70 was mediated through the C-terminal client-binding domain. Moreover, the results in Fig. 2 show that the 2 sub-domains of the client-binding domain no more dimerized.

We here produced a series of $\mathrm{N}$ - and $\mathrm{C}$-terminally truncated forms of the C-terminal 
client-binding domain (Fig. 3a). They were purified to near homogeneity as shown by SDS-PAGE under reducing conditions (Fig. 3b, left). However, upon SDS-PAGE under non-reducing conditions, large-molecular-weight bands (63 or $58 \mathrm{kDa}$ ) appeared for $\mathrm{hHsp} 70$ 382-601 (Fig. 3b, right, lane 3) and hHsp70 382-581 (lane 4). Accompanied by these changes, the 33- and 30-kDa species were decreased in amount. Hence, the high-molecular-mass bands appeared to be dimeric forms mediated through a disulfide bond(s).

We also examined the electrophoretic motility of truncated forms of the client-binding domain on PAGE under non-denaturing conditions (Fig. 3c). The C-terminal truncation of 20 amino acids (hHsp70 382-621) produced smear bands at dimer and trimer regions (lane 2). Successive truncations (hHsp70 382-601 and 382-581) substantially increased the ratio of the slower-migrating species (lanes 3 and 4). Further truncation (hHsp70 382-519) reversed the migration (lane 5).

Next we examined the effect of the N-terminal truncation of the client-binding domain on the dimerization. Eighteen-amino acid truncation (hHsp70 400-641) had little effect (Fig. 3c, lane 6), but further truncations (hHsp70 420-641 and hHsp70 440-641) caused a significant increase in the amount of the slow-migrating species (lanes 7 and 8), corresponding to the dimer. Again, a further truncation (hHsp70 520-641) reversed the mobility (lane 9).

In order to further characterize the molecular configurations, we subjected hHsp70 382-601 to the size-exclusion gel chromatography on Sephacryl S100HR. hHsp70 382-601 was split into 2 peaks (Fig. 4a, top). Polyacrylamide gel electrophoresis under non-denaturing conditions revealed that the dimer and monomer were eluted at the first and second peaks, respectively (Fig. 4a, middle). A trimer was also recognized at fractions 24 and 25 (Fig. 4a, middle). Upon SDS-PAGE under non-reducing conditions, the first peak contained the 63-kDa covalently-associated dimeric species (Fig. 4a, low). Interestingly, the 33-kDa species was also distributed to the first peak, which indicated that this species existed as a dimer, although it did not possess a disulfide bridge.

hHsp70 381-601 was also subjected to 2-dimensional PAGE with the first dimension PAGE under non-denaturing conditions and second dimension SDS-PAGE under non-reducing 
conditions (Fig. 4b). As a result, 5 major species were recognized: a monomer with the molecular mass of $33 \mathrm{kDa} ; 2$ dimers with the molecular mass of 33 or $63 \mathrm{kDa}$; and 2 trimers with 33- or $63-\mathrm{kDa}$ molecular mass. These results demonstrated that the disulfide bridge was not a prerequisite for the dimerization. Noticeably, the dimer connected by the disulfide bridge migrated slightly faster than that without the bridge on PAGE under non-denaturing conditions, indicating the difference in their configurations (Fig. 4b).

One might criticize that these dimers and trimers were artificially formed through nonspecific interactions of the recombinant proteins. In fact, an X-ray crystallographic study on the C-terminal 10-kDa lid sub-domain of rat Hsc70 revealed a packaging of 48 molecules in a unit cell. To address this issue, we performed a mixing experiment of the lysates containing $\mathrm{hHsp} 70$ 382-581 and 382-601. If the dimeric interaction was randomly formed between any free sulfhydryl groups, a heterodimer of the 2 proteins should be formed twice as much as each homo-dimer. As a result, the hetero-dimer was scarcely formed (Fig. 5, an arrow), indicating its specific interactions.

We also performed immunoblotting analyses of truncated forms of $\mathrm{hHsp} 70$ in the bacterial lysates. As a result, small populations of hHsp70 382-641, 382-621, 382-601 and 382-581 possessed a disulfide bridge(s) in the lysates (Fig. 6a, right arrowheads). However, the immunoblotting profile on PAGE under non-denaturing conditions (Fig. 6b) was indistinguishable from that of Coomassie-stained purified proteins (Fig. 3c), which clearly demonstrated that the dimers of hHsp70 were already formed in the lysates even without a disulfide bridge.

Taken together with the results obtained in above studies, we concluded that (i) the dimer-forming activity of hHsp70 resided in the C-terminal domain, (ii) the activity was significantly enhanced by deletion of either $\mathrm{N}$-terminal or C-terminal amino acids $(>=38-40$ residues) of the domain, (iii) neither the client-binding sub-domain (amino acids 382-520) nor the lid sub-domain (amino acids 521-641) was sufficient for dimer formation, (iv) the dimer of the C-terminally truncated forms (hHsp70 382-581 and 382-601) formed disulfide bond(s), and (v) the disulfide bridge was not a prerequisite for the dimerization.. We suspected that free 
sulfhydryl groups of cysteines were located close to each other in the dimeric configuration.

Characterization of the dimeric complex formed by the $N$ - and C-terminal truncations - We investigated the structural integration of the truncated proteins by limited proteolysis. Were the dimeric configurations of the truncated proteins similar to or considerably altered from that of the client-binding domain? As shown in Fig. 7a, the time courses of proteolysis of hHsp70 382-621 and hHsp70 400-641 were similar to each other and to that profile of the client-binding domain (hHsp70 382-641). Further truncations at the C-terminus indicated that the proteolytic profiles of hHsp70 382-601 and 382-581 were similar to each other and relatively resembled that profile of hHsp70 382-641. In contrast, N-terminally-truncated forms (hHsp70 420-641 and hHsp70 440-641) were highly susceptible to trypsin and their profiles were considerably altered from the hHsp70 382-641 profile (Fig. 7a). This finding was ascertained by the results for the concentration-dependent proteolytic profiles of 3 recombinant proteins: The proteolytic profile of hHsp70 382-641 was similar to that of hHsp70 382-601 and the profile of hHsp70 420-641 was obviously altered from that of hHsp70 382-641 or hHsp70 382-601 (Fig. 7b and c).

The results of the limited proteolysis and subsequent $\mathrm{N}$-terminal amino acid sequencing of tryptic fragments (Table 1) were schematically summarized in Fig. 7c. Noticeably, hHsp70 382-641 as well as hHsp70 382-601 was preferentially attacked at the sites in the C-terminal lid sub-domain, but hHsp70 420-641 was preferentially attacked at the sites in the N-terminal client-binding sub-domain (Table 1 and Fig. 7c). Importantly, the alteration of the proteolytic profiles from hHsp70 382-621 to hHsp70 382-601 and that from hHsp70 400-641 to hHsp70 420-641 (Fig. 7) were accompanied by the acquisition of potent dimer-forming activity (Fig. 3). Accordingly, we tentatively classified the truncated proteins into 3 types: (i) hHsp70 382-641, 382-621 and 400-641, which were predominantly monomeric; (ii) hHsp70 382-581 and 382-601, which were predominantly dimeric, and had a configuration similar to that of the first type; and (iii) hHsp70 420-641 and 440-641, which were predominantly dimeric, and had a structure that was considerably altered from those of other types. 
A disulfide bridge(s) of the full-length form of hHsp70 - The C-terminal truncation of the client-binding domain induced efficient conversion from a monomer to a dimer, and the resulting dimer possessed a disulfide bridge(s). The disulfide bridge(s) appeared to be also present in the full-length form of hHsp70 (Fig. 1). For the full-length form, although the population of the species that possessed the disulfide bond appeared to be limited, the ratio in the dimer should be relatively high if the small population of the dimeric species is considered (Fig. 1b). However, there were additional high molecular-mass bands, the subunit composition of which could not be readily estimated. Hence, we performed a truncation experiment on the full-length form similar to that performed on the client-binding domain. We expressed a series of C-terminal truncation forms of hHsp70 (Fig. 8a). Sodium dodecylsulfate-PAGE under non-reducing conditions revealed the existence of a high molecular species (Fig. 8b, left, lanes 1-3). Moreover, in consistent to the result on the client-binding domain (Fig. 3b), C-terminal truncation of 40 or 60 amino acids caused the dimer formation more efficiently (Fig. 8b, left, lanes 2 and 3). Noticeably, the higher molecular species of respective proteins migrated slowest in the full-length form (lane 1); moderately in hHsp760 1-601 (lane 2); and fastest in hHsp70 1-581 (lane 3), reflecting their molecular masses. This finding confirms that the high-molecular species of the full-length form migrating at 150-kDa position was a dimer of $\mathrm{hHsp} 70$ mediated through a disulfide bridge(s). The recombinant protein deleting its C-terminal 80 amino acids (hHsp70 1-561) completely lost the bridge (lane 4).

We subjected recombinant proteins to size exclusion chromatography on Sephacryl S300 (Fig. 8c). Under the conditions employed, monomeric species were eluted at fractions 46-48. A gradual retardation in migration from fraction 46 for $\mathrm{hHsp} 70$ 1-641 and hHsp70 1-581 to fraction 48 for hHsp70 1-561 and hHsp70 1-541 should reflect successive C-terminal truncations. The full length form was mainly eluted as a monomer; and a small population around fraction 40 , the position of the dimer (Fig. 8c). In contrast, hHsp70 1-581 and hHsp70 1-561 were split into 2 peaks, i.e., dimer (fractions 40-42) and monomer (fractions 46-48). The first peak of hHsp70 1-581 migrated to the 140-kDa position on the SDS-PAGE gel under non-reducing conditions, but that of hHsp70 1-561 did so to the 60-kDa position, indicating that the hHsp70 1-561 dimer did 
not possess a disulfide bridge. hHsp70 1-541 (Fig. 8c) and hHsp70 1-520 (data not shown) were eluted as a monomer.

Taken together, these findings indicate that efficient formation of an $\mathrm{hHsp} 70$ dimer and disulfide bond(s) induced by $\mathrm{C}$-terminal truncation was not specific to the client-binding domain, but also occurred in the case of the full-length form. Importantly, hHsp70 1-561 formed a dimer, although it did not contain a disulfide bond. This finding confirmed that the disulfide bridge is not a prerequisite for the dimer formation.

\section{DISCUSSION}

In the present study, we investigated the dimeric structure of $\mathrm{hHsp} 70$. Although a small population of $\mathrm{hHsp} 70$ is present as a dimer, the dimer was more efficiently formed when the C-terminal amino acids (40-80 residues) were deleted from the client-binding domain as well as the full-length form. Similarly, Ohno et al. (29) reported that mouse Hsp70 1-615, from which the $\mathrm{C}$-terminal 26 residues had been deleted tended to form dimer and oligomers more efficiently than did the full-length form. There are only 2 cysteine residues, i.e., Cys574 and Cys603, in the client-binding domain of hHsp70. Because hHsp70 382-581 as well as hHsp70 382-601, in which Cys603 was lost, formed a disulfide bridge, it is reasonable to conclude that Cys574 of a molecule was associated with Cys574 of another molecule in a dimer. In fact, hHsp70 1-561, of which a half population still formed a dimer, did not form a disulfide bridge (Fig. 8c).

Although the N-terminal deletion of the client-binding domain also induced the dimer formation, the configuration of the resulting dimer was considerably distinct from that of the dimer of the client-binding domain, because the client-binding domain with a truncated $\mathrm{N}$-terminus was highly susceptible to proteolysis. The N-terminal sequencing analysis of the proteolytic fragments confirmed this notion, because peptide bonds (Arg469-Gly470 and Arg517-Met518) located within the client-binding sub-domain became susceptible to trypsin by N-terminal truncation of the domain (Table 1). In contrast, the dimer formed by the truncation from the C-terminus showed proteolytic profiles similar to the profile of the client-binding domain, indicating their structural 
integrity. Moreover, we found the presence of a trimer of hHsp70 (Fig. 1). A trimer was also recognized on hHsp70 382-641, 382-601 and 382-581, but not on hHsp70 420-641 and 440-641 (Figs. 3 and 4), which further suggested the similarity of the dimeric structure of the full-length form to those of hHsp70 382-641 and its C-terminally-truncated molecules.

Previous studies consistently showed that the C-terminal client-binding domain is responsible for the dimer/oligomer formation of Hsc70 (19). However, there was an apparent discrepancy as to the role of sub-domains, whether the client-binding sub-domain or the lid sub-domain is critical for this role. The 10-kDa C-terminal lid sub-domain (amino acids 542-646) of rat Hsc70, which forms an elongated bundle-like structure, dictates the self-association (22). In contrast, Fouchaq et al. (20) reported that the 17-kDa peptide-binding sub-domain (amino acids 385-540 of Hsc70) was involved in the oligomerization process. The results obtained in the present study do not accord with either of these reports. Figure 3 showed that either one of the sub-domains, i.e., the client-binding sub-domain or the lid sub-domain, did not form a dimer but that the client-binding domain partially truncated from either the $\mathrm{N}$ - or $\mathrm{C}$-terminus could form dimers. We also demonstrated that substantial changes in the configuration if the N-terminal segment of the client-binding sub-domain was lost, strongly suggesting that the client-binding sub-domain is essential for dimer formation. Hence, we propose that the client-binding sub-domain (amino acids 382-519) is a prerequisite but that an adjacent region (amino acids 520-561 of the lid sub-domain) is indispensable for the dimerization.

Chou et al. (22) reported the crystal structure of the C-terminal 10-kDa lid sub-domain of rat Hsc70. The lid sub-domain formed a package of 48 molecules in a unit cell. In the structure, the association was mediated by the coiled coil-like dimers in an anti-parallel manner, and then the dimers formed a cruciform tetrameric structure in an asymmetric manner. However, the lid sub-domain could not form a dimer under our conditions, and the deletion of more than 38 amino acid residues from the N-terminus of the client-binding domain (residues 382-641) produced a dimer with the conformation that was distinct from that of the client-binding domain. These findings strongly suggest that the oligomeric configuration formed by the lid sub-domain is distinct from that of the native dimer. According to the structure proposed by Chou et al. (22), the lid 
sub-domain of one molecule associates with that of another molecule in an anti-parallel manner, in which 2 cysteines are separated from each other, and therefore, do not seem to form a disulfide bridge. Thus, we insist that the dimeric structure proposed by Chou et al. (22) should be carefully re-investigated.

At last, we propose the mechanism on the dimerization and the formation of the disulfide bridge mediated through Cys574 as follows: Hsp70 could form a dimer through the C-terminal client-binding domain. In a dimeric structure, Cys574 of an Hsp70 molecule is located close to Cyts574 of another molecule in a dimer, but the distance is not close enough for the efficient formation of the disulfide bridge. When the C-terminal 40-60 amino acids are deleted, the two cysteine residues could access to each other presumably because of the destabilization of the C-terminal domain. This is likely to be the case, because the formation of the bridge resulted in a conversion of an hHsp70 dimer into a different configuration as shown in Fig. 4b: The dimer of hHsp70 382-601 that possessed the disulfide bridge had a configuration migrating faster on PAGE under non-denaturing conditions than that without the bridge did (Fig. 4b). This finding strongly suggests that the dimer with the disulfide bridge has a more compact configuration that that without the bridge. A further study on the dimeric structure of hHsp70 is under going in our laboratory.

We greatly acknowledge Mr. Takeshi Kobayakawa for his technical assistance. 


\section{REFRENCES}

1. Xu, Z., Horwich, A., L., and Sigler, P.B. (1997) The crystal structure of the asymmetric GroEL-GroES-(ADP) 7 chaperonin complex. Nature 388, 741-750.

2. Minami, Y., Kimura, Y., Kawasaki, H., Suzuki, K., \& Yahara, I. (1994) The carboxy-terminal region of mammalian HSP90 is required for its dimerization and function in vivo. Mol. Cell. Biol. $14,1459-1464$.

3. Nemoto, T., Ohara-Nemoto, Y., Ota, M., Takagi, T., \& Yokoyama, K. (1995) Mechanism of dimer formation of the 90-kDa heat-shock protein. Eur. J. Biochem. 233, 1-8.

4. Prodromou, C., Roe, S. M., O’Brien, R., Ladbury, J. E., Piper, P. W., \& Pearl, L. H. (1997) Identification and structural characterization of the ATP/ADP-binding site in the Hsp90 molecular chaperone. Cell 90, 65-75.

5. Prodromou, C., Roe, S. M., Piper, P. W., \& Pearl, L. H. (1997) A molecular clamp in the crystal structure of the N-terminal domain of the yeast Hsp90 chaperone. Nature Struct. Biol. 4, 477-482.

6. Stebbins, C. E., Russo, A. A., Schneider, C., Rosen, N., Hartl, F. U., \& Pavletich, N. P. (1997) Crystal structure of an Hsp90-geldanamycin complex: targeting of a protein chaperone by an antitumor agent. Cell 89, 239-250.

7. Meyer, P., Prodromou, C., Hu, B., Vaughan, C., Roe, S.M., Panaretou, B., Piper, P.W., \& Pearl, L.H. (2003) Structural and functional analysis of the middle segment of Hsp90: implications for ATP hydrolysis and client protein cochaperone interactions. Mol. Cell 11, 647-658.

8. Welch, W. J. \& Feramisco, J. R. (1982) Purification of the major mammalian heat shock proteins. J. Biol. Chem. 257, 14949-14959.

9. Palleros, D. R., Reid, K. L., Shi, L., \& Fink, A. L. (1993) DnaK ATPase activity revisited. FEBS lett. 336, 124-128.

10. Blond-Elguindi, S., Fourie, A. M., Sambrook, J. F., \& Gething, M.-J., H. (1993) Peptide-dependent stimulation of the ATPase activity of the molecular chaperone BiP is the result of conversion of oligomers to active monomers. J. Biol. Chem. 268, 12730-12735.

11. Azem, A., Oppliger, W., Lustig, A., Jeno, P., Feifel, B., Schatz, G., \& Horst, M. (1997) The mitochondrial hsp70 chaperone system: effect of adenine nucleotides, peptide substrate, and 
mGrpE on the oligomeric state of mhsp70 J. Biol. Chem. 272, 20901-20906.

12. Kim, D., Lee, Y. J., \& Corry, P. M. (1992) Constitutive HSP70: oligomerization and its dependence on ATP binding. J. Cell Physiol. 153, 353-361.

13. Chappell, T. G., Konforti, B. B., Schmid, S. L., \& Rothman, J. E. (1987) The ATPase core of a clathrin uncoating protein. J. Biol. Chem. 262, 746-751.

14. Stevens, S. Y., Cai S., Pellecchia, M., \& Zuiderweg, E. R. P. (2003) The solution structure of the bacterial HSP70 chaperone protein domain DnaK (393-507) in complex with the peptide NRLLLTG Prot. Sci. 12, 2588-2596.

15. Burkholder, W. F., Zhao, X., Zhu, X., Hendrickson, W. A., Gragerov, A., \& Gottesman, M. E. (1996) Mutations in the C-terminal fragment of DnaK affecting peptide binding. Proc. Natl. Acad. Sci. U.S.A. 93, 10632-10637.

16. Takeda, S., \& McKay, D. B. (1996) Kinetics of peptide binding to the bovine $70 \mathrm{kDa}$ heat shock cognate protein, a molecular chaperone. Biochemistry 35, 4636-4644.

17. Wang, C., \& Lee M. R. (1993) High-level expression of soluble rat hsc70 in Escherichia coli: purification and characterization of the cloned enzyme. Biochem. J. 294, 69-77.

18. Flynn, G. C., Chappell, T. G, \& Rothman, J. E. (1989) Peptide binding and release by proteins implicated as catalysts of protein assembly. Science 245, 385-390.

19. Benaroudj, N., Fouchaq, B., \& Ladjimi, M. M. (1997) The COOH-terminal peptide binding domain is essential for self association of the molecular chaperone HSC70. J. Biol. Chem. 272, 8744-8751.

20. Fouchaq, B., Benaroudj, N., Ebel, C., \& Ladjimi, M. M. (1999) Oligomerization of the 17-kDa peptide-binding domain of the molecular chaperone HSC70. Eur. J. Biochem. 259, 379-384.

21. Wang, T. F., Chang, J.-H., \& Wang, C. (1993) Identification of the peptide binding domain of hsc70: 18-kilodalton fragment located immediately after ATPase domain is sufficient for high affinity binding. J. Biol. Chem. 268, 26049-26051.

22. Chou, C.-C., Forouhar, F., Yeh, Y.-H., Shr, H.-L., Wang, C., \& Hsiao, C.-D. (2003) Crystal structure of the C-terminal 10-kDa subdomain of Hsc70. J. Biol. Chem. 278, 30311-30316.

23. Hunt, C., \& Morimoto, R. I. (1985) Conserved features of eukaryotic hsp90 genes revealed by 
comparison with the nucleotide sequence of human hsp70. Proc. Natl. Acad. Sci. U.S.A. 82, 6455-6459.

24. Milner, C. M., \& Campbell, R. D. (1990) Structure and expression of the three MHC-linked HSP70 genes. Immunogenetics 32, 242-251.

25. Laemmli, U. K. (1970) Cleavage of structural proteins during the assembly of the head of bacteriophage T4. Nature (London) 227, 680-685.

26. Nishihara, J., Ishibashi, T., Sakai, M., Nishi, S., Kumazaki, T., Hatanaka, Y., Tsuda, S., \& Hikichi, K. (1992) Characterization of cysteine residues of glutathione $S$-transferase P: evidence for steric hindrance of substrate binding by a bulky adduct to cysteine 47. Biochem. Biophys. Res. Commun. $188,424-432$.

27. Nemoto, T., Ota, M., Ohara-Nemoto, Y., \& Kaneko, M. (1995) Identification of dimeric structure of proteins by use of the glutathione $S$-transferase-fusion expression system. Anal. Biochem. 227, 396-399.

28. Nemoto, T., \& Sato, N. (1998) Oligomeric forms of the 90-kDa heat shock protein. Biochem. J. 330, 989-995.

29. Ohno, M., Kitabatake, N., \& Tani, F. (2004) Role of the C-terminal region of mouse inducible Hsp72 in the recognition of peptide substrate for chaperone activity. FEBS Lett. 576, 281-286. 
Table 1. N-terminal sequences of tryptic fragments of truncated hHsp70.

Origin Fragment $(\mathrm{kDa}) \quad$ Amino acids detected by sequencing Corresponding sequence

\begin{tabular}{|c|c|c|c|}
\hline \multirow[t]{8}{*}{$382-641$} & 35 (intact) & n. d & \\
\hline & 33 & GGSHHHHH & $\mathrm{m}_{35} \mid \mathrm{g}_{-34} \mathrm{gshhhhh}$ \\
\hline & 30 & DLYDDDD & $\mathrm{r}_{-13} \mid \mathrm{g}_{-12} \mathrm{lydddd}$ \\
\hline & 25 & DLYDDDDKDP & $\mathrm{r}_{-13} \mid \mathrm{g}_{-12} 1 \mathrm{yddddkdp}$ \\
\hline & $21^{\mathrm{a}}$ & GVPQIEVTFD & $\mathrm{R}_{469} \mid \mathrm{G}_{470} \mathrm{QIEVTFD}$ \\
\hline & & MVQEAEKYKA & $\mathrm{R}_{517} \mid \mathrm{M}_{518} \mathrm{QEAEKYKA}$ \\
\hline & 15 & GVPQIEVT (major) & $\mathrm{R}_{469} \mid \mathrm{G}_{470} \mathrm{QIEVTFD}$ \\
\hline & 14 & GVPQIEVT (major) & $\mathrm{R}_{469} \mid \mathrm{G}_{470} \mathrm{QIEVTFD}$ \\
\hline \multirow[t]{3}{*}{$382-601$} & 33 (intact) & n. d. & \\
\hline & 27 & DYLDDDDKD & $\mathrm{r}_{-13} \mid \mathrm{g}_{-12}$ lyddddkd \\
\hline & 25 & GGSHHHHHH & $\mathrm{m}_{-35} \mid \mathrm{g}_{-34} \mathrm{gshhhhhh}$ \\
\hline \multirow[t]{4}{*}{$420-641$} & 33 (intact) & n. d. & \\
\hline & 23 & GVPQIEVTFD & $\mathrm{R}_{469} \mid \mathrm{G}_{470} \mathrm{VPQIEVTFD}$ \\
\hline & $21^{\mathrm{a}}$ & GVPQIEVTFD & $\mathrm{R}_{469} \mid \mathrm{G}_{470} \mathrm{VPQIEVTFD}$ \\
\hline & & MVQEAEKYKA & $\mathrm{R}_{517} \mid \mathrm{M}_{518} \mathrm{VQEAEKYKA}$ \\
\hline
\end{tabular}

Deduced sequences written in capital and small letters were derived from $\mathrm{hHsp} 70$ and the tagged sequence, respectively. $\quad{ }^{a}$ a mixture of 2 fragments. $\quad{ }^{b} \mathrm{~N}$-terminal sequence of the major species was determined. n.d., not determined. 


\section{FIGURE LEGENDS}

Fig. 1. Dimerization of the full-length form of hHsp70. The full-length form of recombinant hHsp70 was electrophoresed on SDS-PAGE at a 7.5\% polyacrylamide concentration under non-reducing and reducing conditions (a) or on PAGE at a 7.5\% polyacrylamide concentration under non-denaturing conditions (b). Lane 1, $0.3 \mu \mathrm{g}$; lane 2, $1 \mu \mathrm{g}$; lane 3, $3 \mu \mathrm{g}$; and lane 4, $10 \mu \mathrm{g}$. Molecular markers (Mk) used are low-molecular-weight markers and kaleidoscope markers (a) and markers for native PAGE. M, monomer; $\mathrm{D}$, dimer and T, trimer.

Fig. 2. Dimerization of the client-binding domain and its truncated forms. (a) The ATP-binding domain (amino acids 1-381), the client-binding domain (amino acids 382-641) and 2 sub-domains of the client-binding domain are schematically illustrated. (b) Recombinant proteins were separated by SDS-PAGE under reducing conditions (left) or by PAGE under non-denaturing conditions (right). Protein samples loaded on SDS-PAGE under reducing conditions $(0.5 \mu \mathrm{g})$ and on PAGE under non-denaturing conditions $(1.5 \mu \mathrm{g})$ in lanes 1-5 correspond to numbers 1-5 of panel "a". Mk, molecular markers. Arrowheads indicate minor species migrating slower than the major species (right, lanes 1, 3 and 5). An asterisk indicates a 22-kDa band (see text). (c) hHsp70 1-381 (1.3 mg/2 ml) was separated by size-exclusion gel chromatography on a Sephacryl S100HR column (upper panel) as described in "EXPERIMEMENTAL PROCEDURES." Ovalbumin ( $45 \mathrm{kDa}$, Stokes' radius $=30.5 \mathrm{~A}$ ) was subjected to the column separately as a size reference. hHsp70 1-381 (lane P, $1.5 \mu \mathrm{g}$ ) or aliquots (10 $\mu \mathrm{l}$ ) of fractions 27-36 of the chromatography were separated by SDS-PAGE under non-reducing conditions (middle panel) or by PAGE under non-denaturing conditions (lower panel). (d and e) Twenty-five $\mu \mathrm{g}$ of hHsp70 382-641 (d) and 520-641 (e) were separated by 2-dimenisional PAGE as described in "EXPERIMENTAL PROCEDURES." The second dimension SDS-PAGE was performed under reducing conditions. Apparent molecular masses of recombinant proteins are indicated on the right. Asterisks indicate a $22-\mathrm{kDa}$ band (see text). $\mathrm{M}, \mathrm{D}, \mathrm{T}$ and $\mathrm{O}$ represent monomer, dimer, trimer and oligomer, respectively. 
Fig. 3. Dimerization of the client-binding domain and its truncated forms. (a) The client-binding domain (amino acids 382-641) and its truncated forms are presented. States of the proteins $(\mathrm{M}$, monomer and $\mathrm{D}$, dimer) and the formation of disulfide bridges (S-S) observed in panels "b" and "c" are summarized on the right. Recombinant proteins were separated by SDS-PAGE under reducing and non-reducing conditions (b) or by PAGE under non-denaturing conditions (c). Protein samples loaded on SDS-PAGE $(0.5 \mu \mathrm{g})$ and on PAGE under non-denaturing conditions (1.5 $\mu \mathrm{g})$ in lanes 1-9 of panels "b" and "c," respectively, correspond to numbers 1-9 of panel "a". Mk, molecular markers. M, D and T on the left of panel "c" represent monomer, dimer and trimer, respectively.

Fig. 4. Size-exclusion gel chromatography and 2-dimensional PAGE of hHsp70 382-601. (a) hHsp70 382-601 (3 mg/2 ml) was separated by size-exclusion gel chromatography on a Sephacryl S100HR column (upper panel) as described in "EXPERIMEMENTAL PROCEDURES.” Size markers [Blue dextran 2000 (void volume), bovine serum albumin (66 kDa, Stokes' radius=35.5 A), and ovalbumin ( $45 \mathrm{kDa}$, Stokes' radius $=30.5 \mathrm{~A})$ ] were subjected to the chromatography separately. An aliquot $(7.5 \mu \mathrm{l})$ of each fraction was subjected to PAGE under non-denaturing conditions (middle panel) or SDS-PAGE under non-reducing conditions (lower panel). P, purified hHsp70 382-601 loaded on the chromatography column. Mk, molecular markers. M, $\mathrm{D}$ and $\mathrm{T}$ on the left represent monomer, dimer and trimer, respectively. (b) Two-dimensional PAGE of hHsp70 382-601 (20 $\mu \mathrm{g})$. As references of first and second dimensional PAGE, hHsp70 382-601 (2 $\mu \mathrm{g})$ was run in parallel. Apparent molecular masses of recombinant proteins are indicated on the right. Mk, marker proteins. $\mathrm{M}, \mathrm{D}$ and $\mathrm{T}$ represent monomer, dimer and trimer, respectively.

Fig. 5. Time course of the formation of the disulfide bridge(s) - Bacterial lysate containing hHsp70 382-581, hHsp70 382-601 or their equal mixture was prepared. Immediately after the lysate preparation or after 1 or 5 days being kept at $4^{\circ} \mathrm{C}$, the lysates $(1 \mathrm{ml})$ were mixed with Talon affinity resin [50 $\mu \mathrm{l}$ as a $50 \%(\mathrm{v} / \mathrm{v})$ suspension]. After the washing of the resin with washing 
buffer $(1 \mathrm{ml}) 5$ times, bound proteins were extracted with $50 \mu \mathrm{l}$ of $100 \mathrm{mM}$ imidazole (pH 8.0) containing $10 \%(\mathrm{v} / \mathrm{v})$ glycerol. Then, aliquots $(7.5 \mu \mathrm{l})$ were electrophoresed on SDS-PAGE under non-reducing conditions. $\mathrm{M}$, monomer; and $\mathrm{D}$, dimer. $\mathrm{Mk}$, molecular markers. An arrow represents a hetero-dimer.

Fig. 6. Immunoblotting analysis of the client-binding domain and its truncated forms. Bacterial lysates containing recombinant proteins $(2.5 \mu \mathrm{l})$ were subjected to SDS-PAGE under reducing and non-reducing conditions (a) or PAGE under non-denaturing conditions (b). Separated proteins were immunoblotted with an anti-His tag monoclonal antibody as described in "EXPERIMENTAL PROCEDURES." Mk, molecular markers. On PAGE under non-denaturing conditions, markers in the polyacrylamide gel were stained with Coomassie brilliant blue. M, monomer; and $\mathrm{D}$, dimer. Proteins loaded in lanes 1-9 are identical to those of Fig. 3.

Fig. 7. Limited proteolysis of the client-binding domain and its truncated forms. (a) The client-binding domain (hHsp70 382-641) and its truncated forms $(5 \mu \mathrm{g} / 50 \mu \mathrm{l})$ were incubated without trypsin (lane 1) or with $0.2 \mu \mathrm{g}$ TPCK-treated trypsin at $30^{\circ} \mathrm{C}$ for $1.5 \mathrm{~h}$ (lane 2 ) or $10 \mathrm{~h}$ (lane 3). Proteolytic fragments were separated by the Tris-Tricine SDS-PAGE under reducing conditions. M, molecular markers. (b) hHsp70 382-641, hHsp70 382-601 and hHsp70 $420-641(12.5 \mu \mathrm{g} / 50 \mu \mathrm{l})$ were incubated at $30^{\circ} \mathrm{C}$ for $12 \mathrm{~h}$ without (lane 1$)$ or with $3 \mathrm{ng}$ (lane 2), 10 ng (lane 3), $30 \mathrm{ng}$ (lane 4), $0.1 \mu \mathrm{g}$ (lane 5), $0.3 \mu \mathrm{g}$ (lane 6), or $1 \mu \mathrm{g}$ of TPCK-trypsin. Aliquots (5 $\mu \mathrm{g})$ were separated on SDS-PAGE gels under reducing conditions by use of the Tris-Tricine system. Other aliquots were separated in the same way and used for $\mathrm{N}$-terminal amino acid sequencing as described in "EXPERIMENTAL PROCEDURES." $\mathrm{M}$, molecular markers. Apparent molecular masses of major proteolytic fragments are indicated in panel "b." (c) Tryptic fragments of 3 recombinant proteins are schematically arranged based on their profiles and $\mathrm{N}$-terminal sequences. Molecular masses of the fragments were estimated from the results shown in panel "b." The N-terminal amino acids were defined in Table 1. Hatched areas 
represent the 35-amino acid $\mathrm{N}$-terminal tag.

Fig. 8. Dimerization of the full-length form and its C-terminally truncated forms. The full-length form and its C-terminally truncated forms expressed are schematically illustrated (a). Cys574 and Cys603 represent the 2 sole cysteines located within the client-binding domain. The results in terms of disulfide bridge formation (S-S) and the states [monomer (M) or dimer (D)] are summarized on the right. (b) SDS-PAGE under non-reducing (left) and reducing conditions (right). Numbers 1-6 are identical to those of panel "a." Mk, molecular markers. (c) Recombinant proteins $(1 \mathrm{mg} / \mathrm{ml}, 0.8 \mathrm{ml})$ were separated on a Sephacryl $\mathrm{S} 300(1 \times 90 \mathrm{~cm})$ column as described in "EXPERIMENTAL PROCEDURES." Affinity-purified samples (P, $1 \mu \mathrm{g})$ and aliquots $(6 \mu \mathrm{l})$ of fractions $28-50$ of the gel chromatography were separated by SDS-PAGE on a $10 \%$ polyacrylamide gel under non-reducing conditions. Apparent molecular masses of recombinant proteins are indicated on the right. $\mathrm{Mk}$, molecular markers. 
Fig. 1

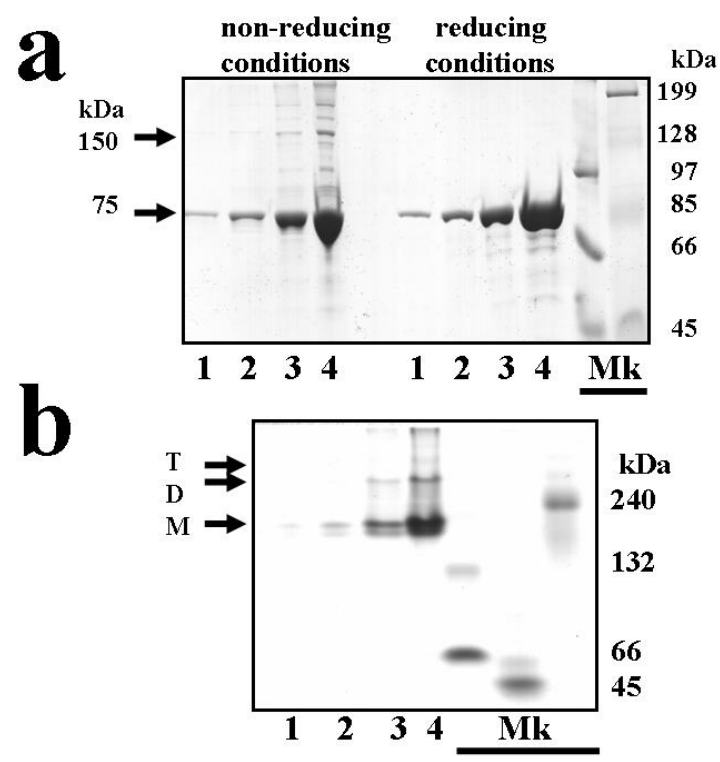

Fig. 2

a

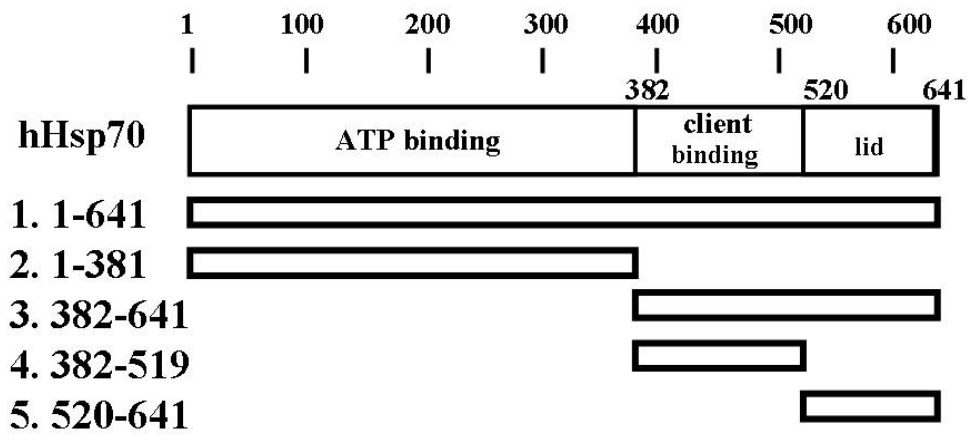


b

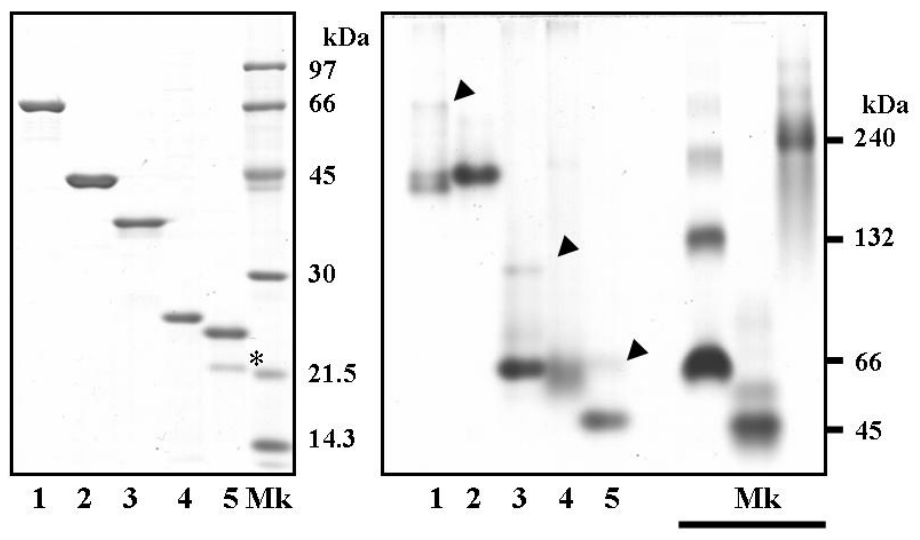

c

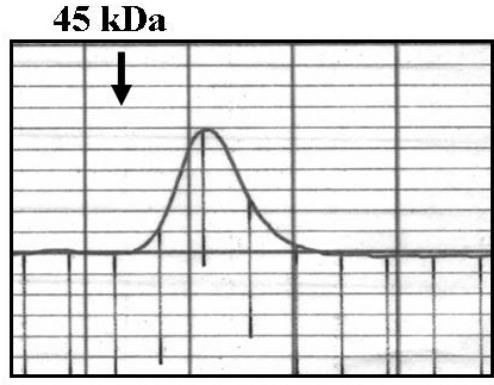

Fr. No. P $27282930313233 \quad 343536$ Mk kDa

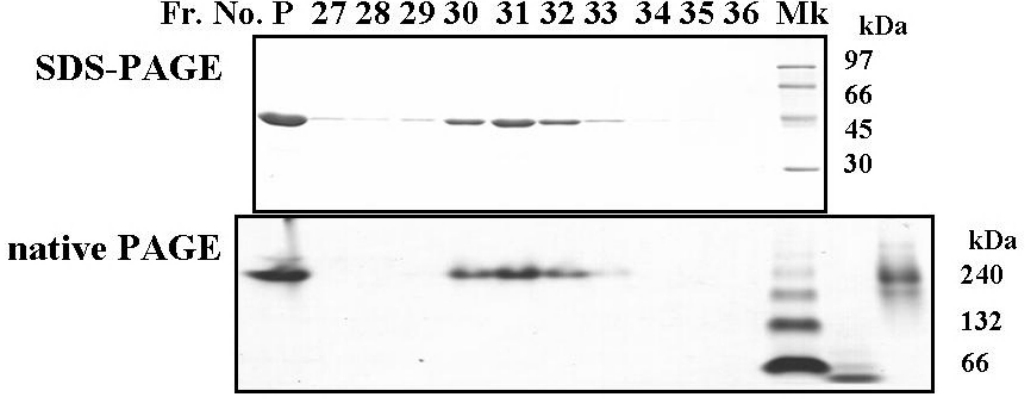



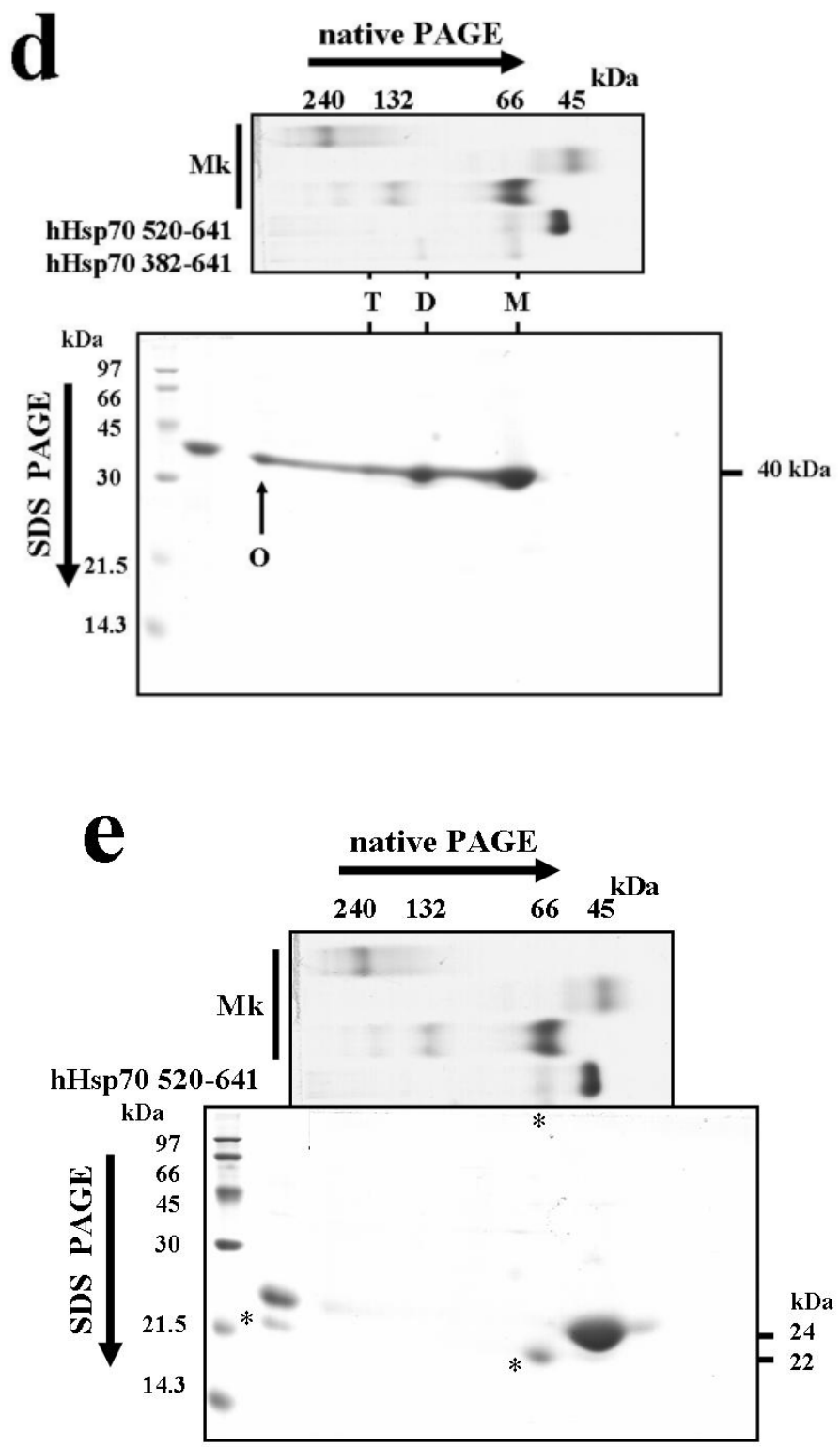
Fig. 3

$\mathbf{a}$

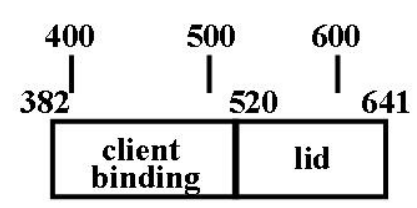

1. 382-641

2. 382-621

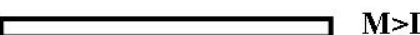

3. 382-601

4. $382-581$

5. 382-519

6. 400-641

7. 420-641

8. 440-641

9. 520-641

$M>D$

$\mathbf{M}>\mathbf{D}$

$\mathbf{M}<<\mathbf{D}(\mathbf{S}-\mathbf{S})$

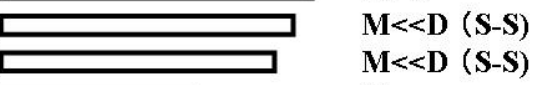

$\square \quad M$

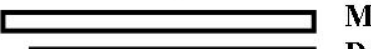

$\square D$

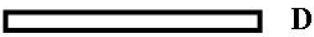
(1)

$\mathbf{M}$

\section{b}

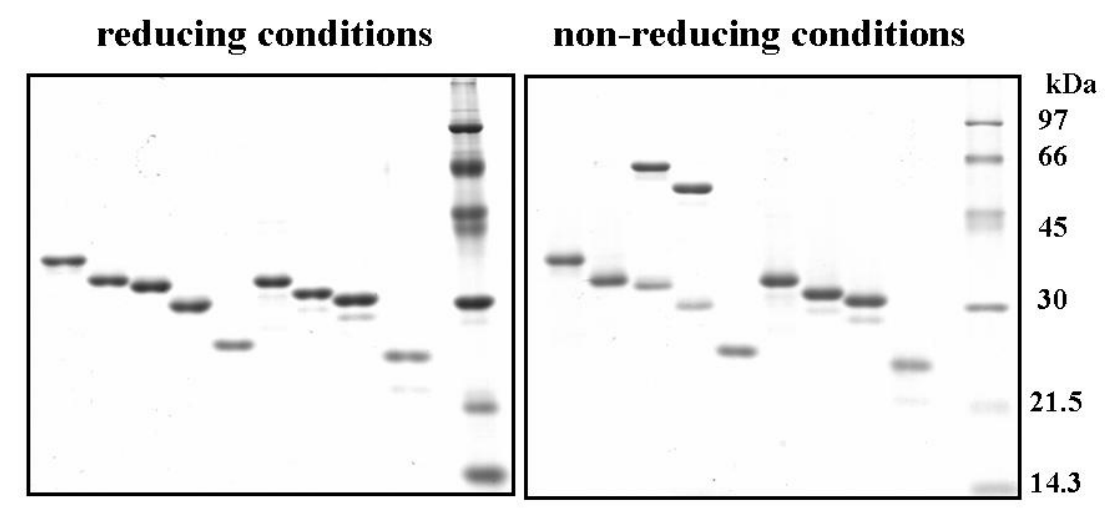

$\begin{array}{lllllllllllllllllllll}1 & 2 & 3 & 4 & 5 & 6 & 7 & 8 & 9 & \text { Mk } & 1 & 2 & 3 & 4 & 5 & 6 & 7 & 8 & 9 & \text { Mk }\end{array}$ 
c

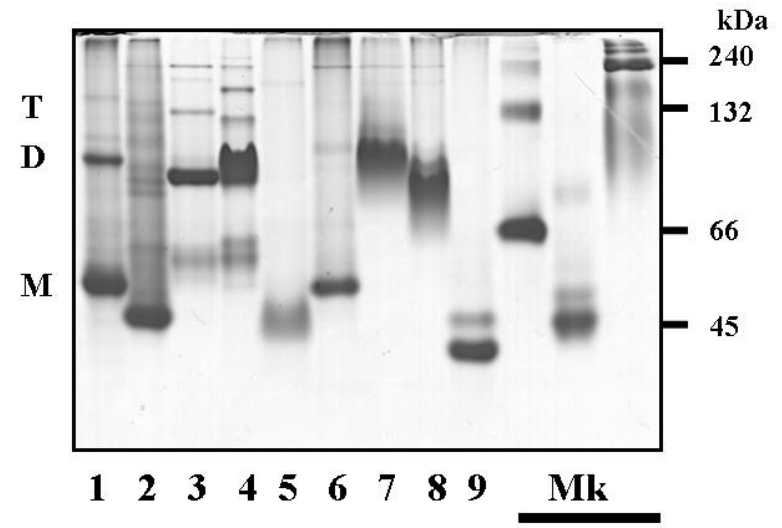

Fig. 4
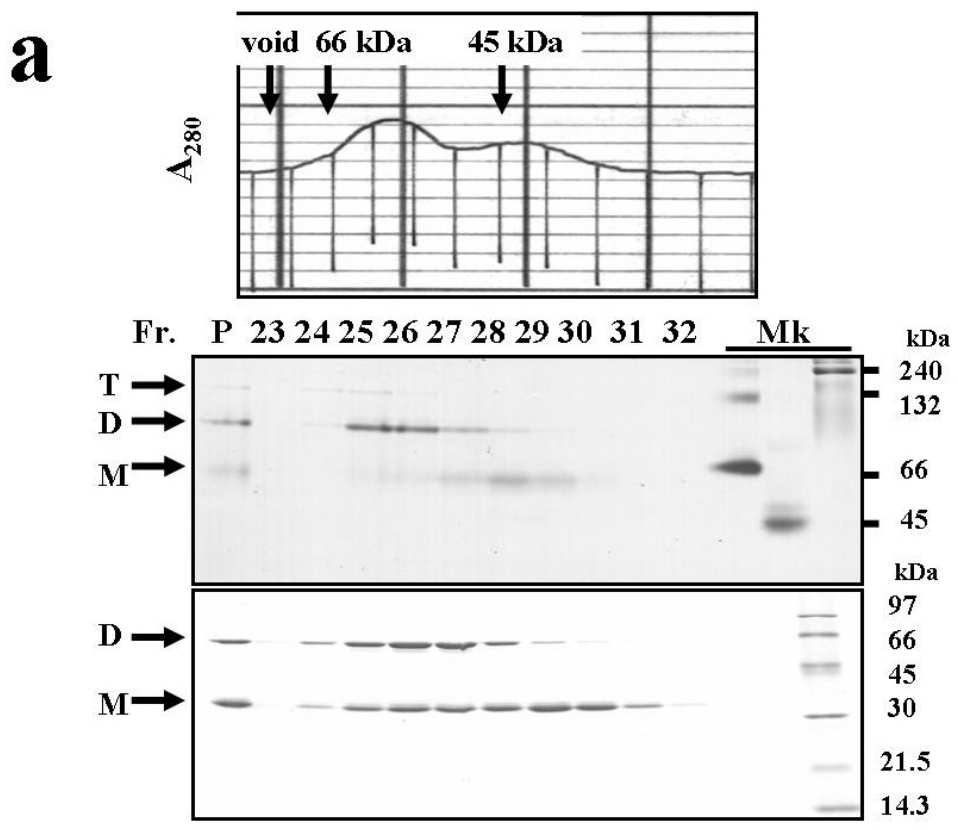


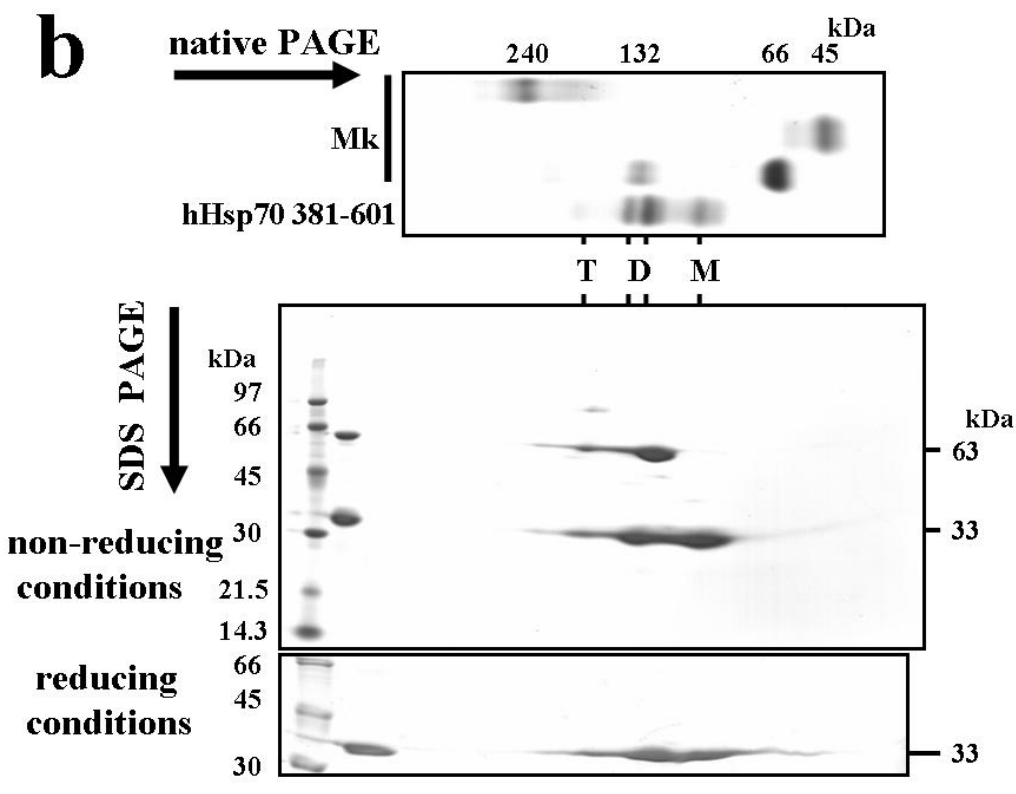

Fig. 5

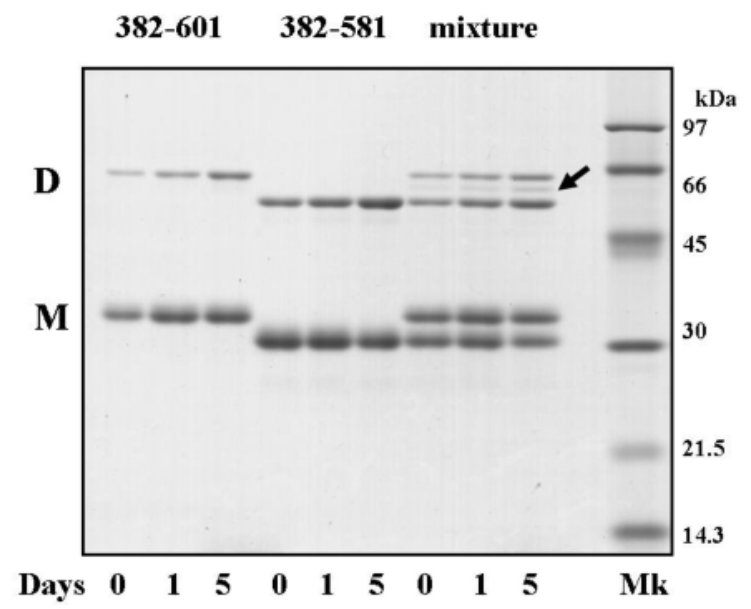


Fig 6

a

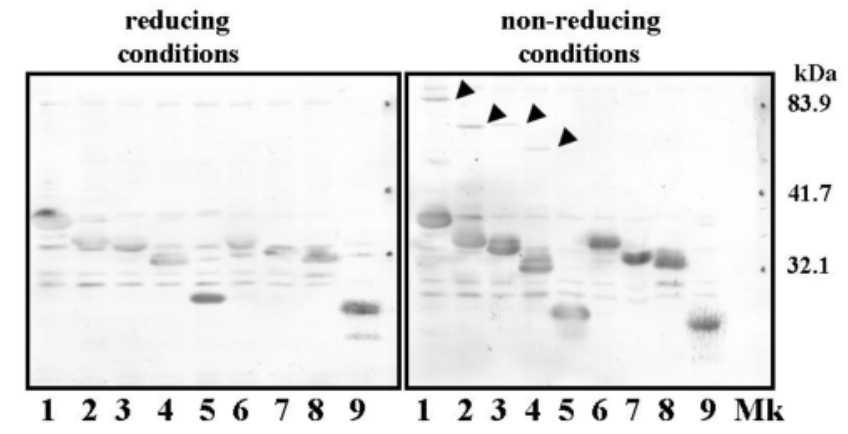

b

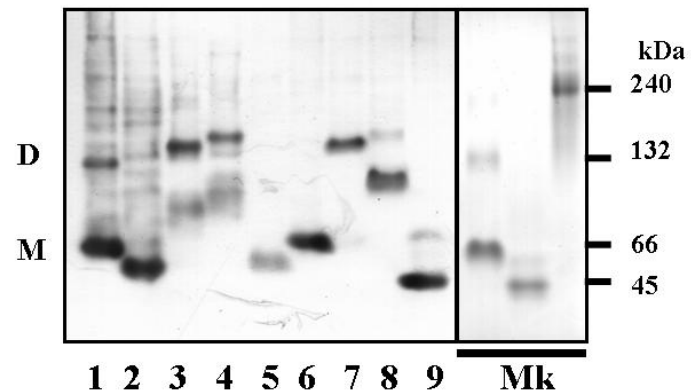


Fig. 7

$\mathbf{a}$

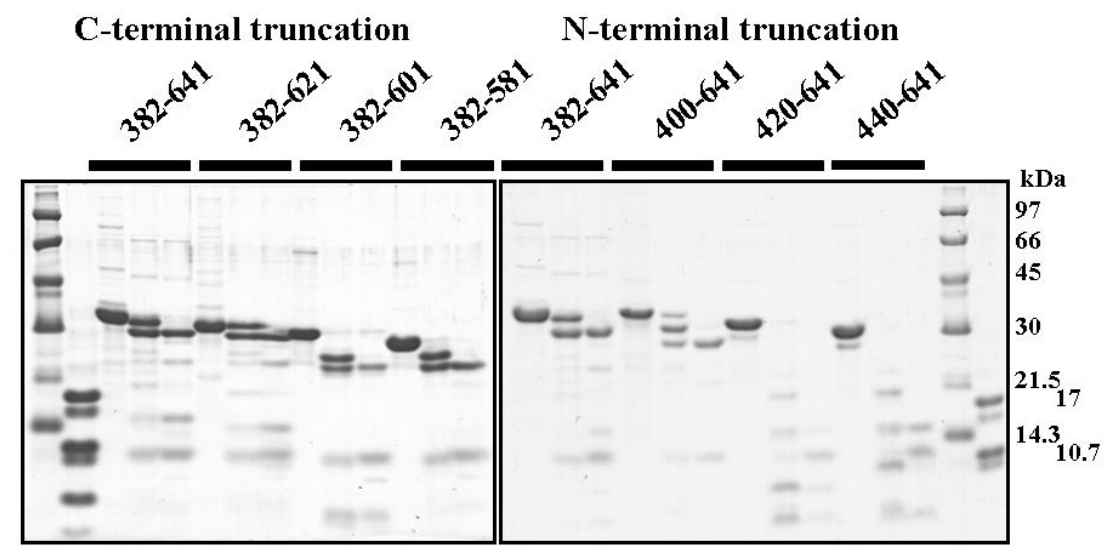

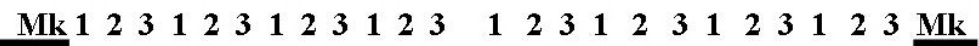

b

$382-641 \quad 382-601$

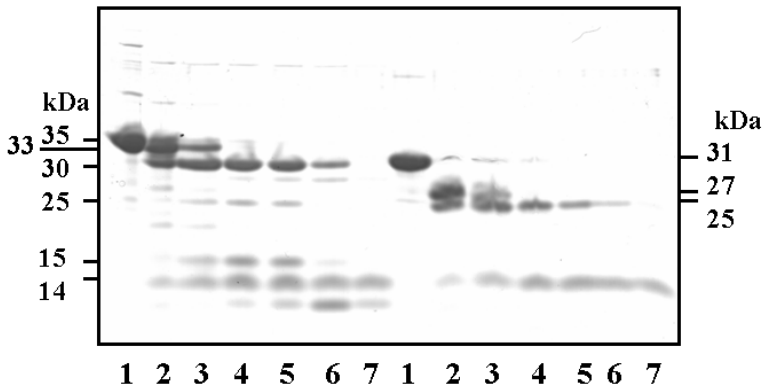




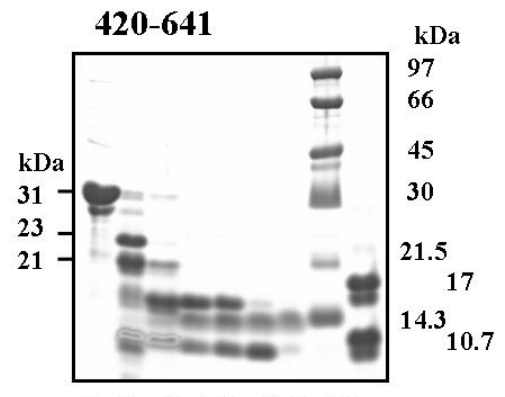

\begin{tabular}{llllllll}
1 & 2 & 3 & 4 & 5 & 6 & 7 & $\mathrm{Mk}$ \\
\hline
\end{tabular}

C

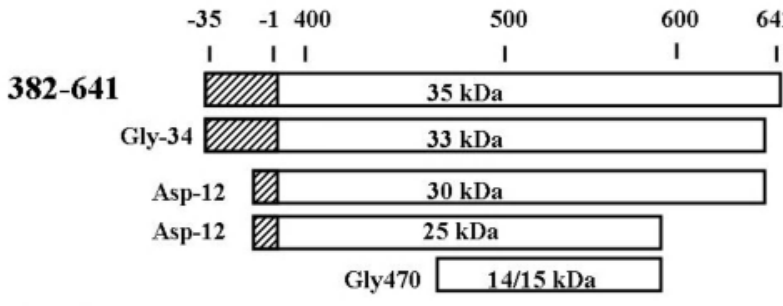

382-601

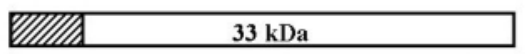

Gly-34 IIIIA $27 \mathrm{kDa}$

Gly-12 $25 \mathrm{kDa}$

420-641

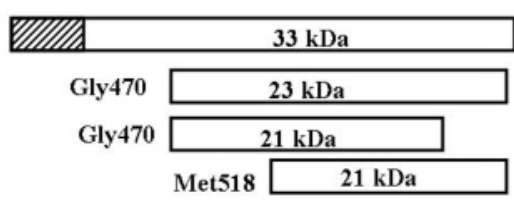


Fig. 8

a

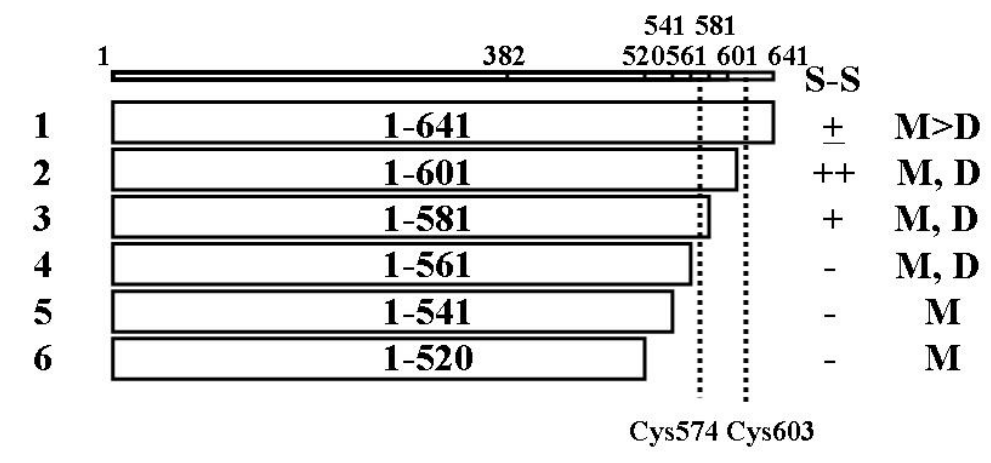

b

non-reducing conditions reducing conditions

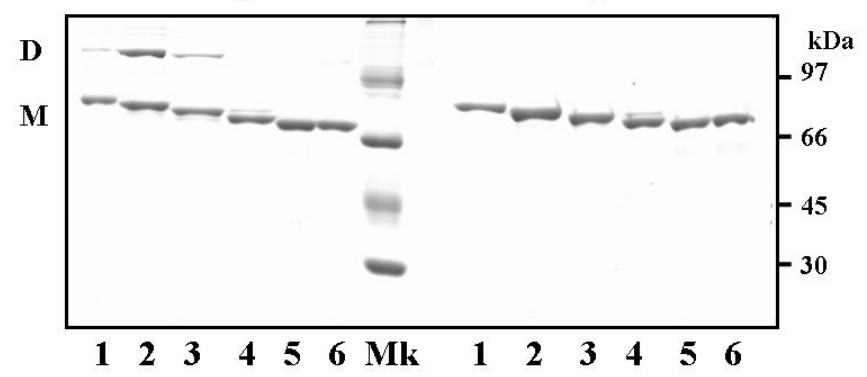




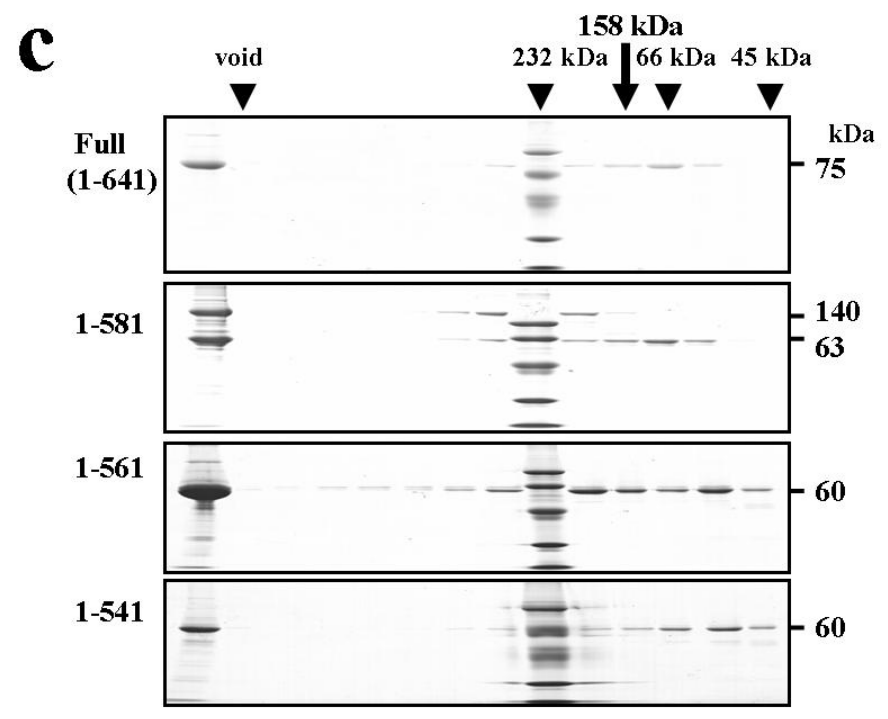

P 28303234363840 Mk 4244464850 